\title{
SHORT-PERIOD SC UNDULATOR ${ }^{1}$ \\ A. Mikhailichenko \\ Cornell University, LEPP, Ithaca NY 14853 \\ Abstract
}

We describe here design of short period helical undulator with SC windings. $2 \mathrm{~mm}$ period, $\mathrm{K} \sim 0.12$ undulator can be used in positron production scheme. Results of test of 6 in-length prototype cold mass is represented also.

\section{INTRODUCTION}

Polarization in future linear collider recognized as a factor increasing collisions rate, effective polarization and as an instrument for drastic reduction of background especially if both electron and positron bunches are polarized. Method for polarized positron production was suggested many years ago [1]. At first stage circularly polarized high-energy gammas generated in a short period helical undulator by (used) high-energy beam after collision. At second stage these circularly polarized gammas converted into positrons/electrons in a thin target. Energy selection of secondary particles delivers polarization to the beam as a result of transferring polarization of gammas to the secondary particles at high-edge of energy spectrum. Polarization with this method can reach $65-70 \%$ with $\sim 130$ m long undulator.

Cold mass of undulator with superconducting coils having period of $1 \mathrm{~cm}, \sim 30 \mathrm{~cm}$-long with $\sim 6$ $\mathrm{mm}$ aperture clearance, was successfully tested in a framework of VLEPP linear collider activity [2], [3]. Field of $\sim 0.5 \mathrm{~T}$ was measured at the axis. The design was recommended for future LC application practically without any changes required.

New growing interest to this subject was indicated at last LC02 [4],[5]. Publication [4] now grew up into proposal for E-166 experiment at SLAC [6], carried by international community. The goal of experiment is to test and develop the concept using SLAC's $50 \mathrm{GeV}$ beam and $\sim 1 \mathrm{~m}$ long short period $(\sim 2 \mathrm{~mm})$ undulator allowing generation of $\sim 10 \mathrm{MeV}$ gammas. One other potential utilization of polarized positrons -arrangement collisions in SLAC B-factory is also connected with undulator-based positron generation. Here $\sim 4-\mathrm{m}$ long undulator allow generation necessary amounts for normal operation of B-Factory [7].

Although right now pulsed undulator recognized as mostly guaranteed and cheap enough for this kind of experiment, SC can be used in future Linear Collider full-scale project. So as it can bee seen as a future possibility, we decided to describe it in a little bit more details.

Basically advantages of undulator with superconducting wires more brightly exposed for working at high-energy primary beam, such as $\geq 200 \mathrm{GeV}$. At this energy the probability of the wall exposure is small. SC undulator also allows operation with (relatively) large aperture about 6-8mm drastically reducing wall exposure. Period about 8-10 $\mathrm{mm}$ gives good safety operational margins. Nice advantage of $\mathrm{SC}$ undulator is that its functionality does not depend on repetition rate.

Main point of concern for small aperture undulator is operational stability of SC coil while few $\mathrm{kW}$ high-energy beams is running within $2 \mathrm{~mm}$ from cold walls. So in any case testing of SC undulator section a prior to final approval will include test undulator under real conditions with

\footnotetext{
${ }^{1}$ Electronic version is available at http://www.Ins.cornell.edu/public/CBN/2003/CBN03-4/CBN03_4.pdf
} 
high-energy beam inside aperture. So in this case manufacturing and testing section of SC undulator is necessary in any case.

For successful operation of conversion system, undulator-having $\geq 1-1.3 \times 10^{4}$ periods required. In any case this undulator will be manufactured as relatively short sections of $\sim 2-\mathrm{m}$ long.

\section{CRYOSTAT}

Cryostat serves as housing for cold mass core. It must keep the cold mass below critical temperature for SC wire and have low heat losses. In addition, as the aperture is small it must provide good geometrical stability of axes positioning. The cryostat sketch is represented in Fig.1. The cryostat has extra features allowing it to operate either with cryocooler and/or with liquid Helium coolant. In future linear collider 100-m long undulator, naturally can be feed by flowing Helium as the total heat losses can be estimated $\sim 100 \mathrm{~W}$.

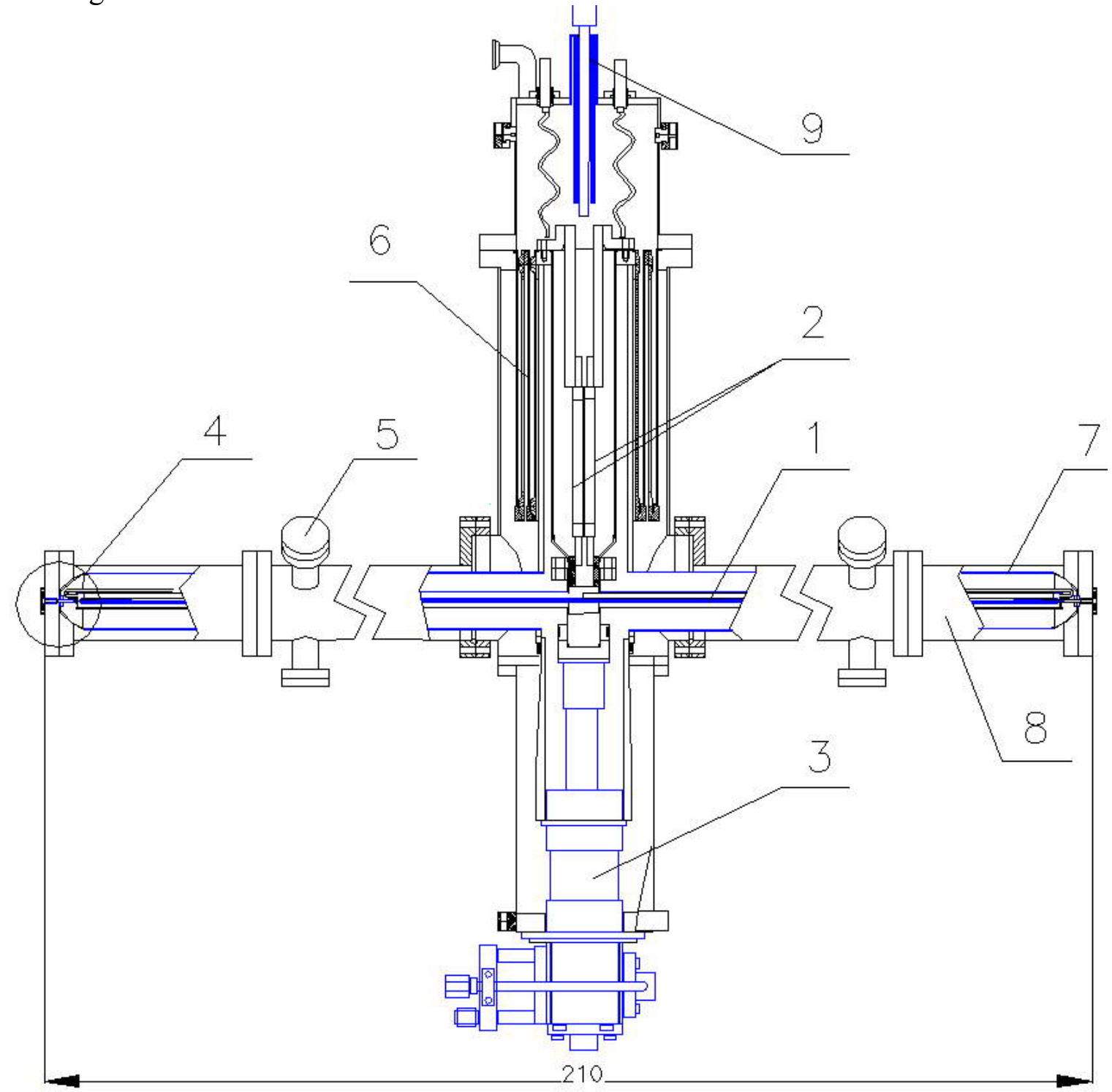

FIGURE 1: Cryostat for 2 m-long module. 1-is a cold mass, 2-HTS leads, 3-Cryocooler, 4-end region, see Fig.2, 5-is suspension elements, Fig.2, 6-are coaxial Al-StSteel cylinders, 7-is intermediate $50^{\circ} \mathrm{K}$ temperature shield, 8-is a vacuumed corps, 9-is filling tubing for operation with filled liquid He. All responsible spaces interlaced by superinsulation. Full height of cryocooler is 21.91 ". 
So, basically, this is a device for testing slightly different concepts of cooling. 2-meter long module will be able to work with cryocooler having $1.5 \mathrm{~W}$ power at LHe temperature and $\sim 40 \mathrm{~W}$ at $50^{\circ} \mathrm{K}$. It also allows operation with filling by LHe directly trough the input 9 in fig. 1 . In this case cryocooler can operate too or can be replaced by LN cylindrical container.

Main support of long cold mass, having $\sim 1^{\prime \prime}$ in diameter and length $\sim 4 \mathrm{~m}$, is going with suspension elements shown in Fig.2. As the cold mass is light weighted this solution allows good control of position of axis. End flanges have adjustable positioning so they will be attached with necessary accuracy after the suspension straps are stretched.
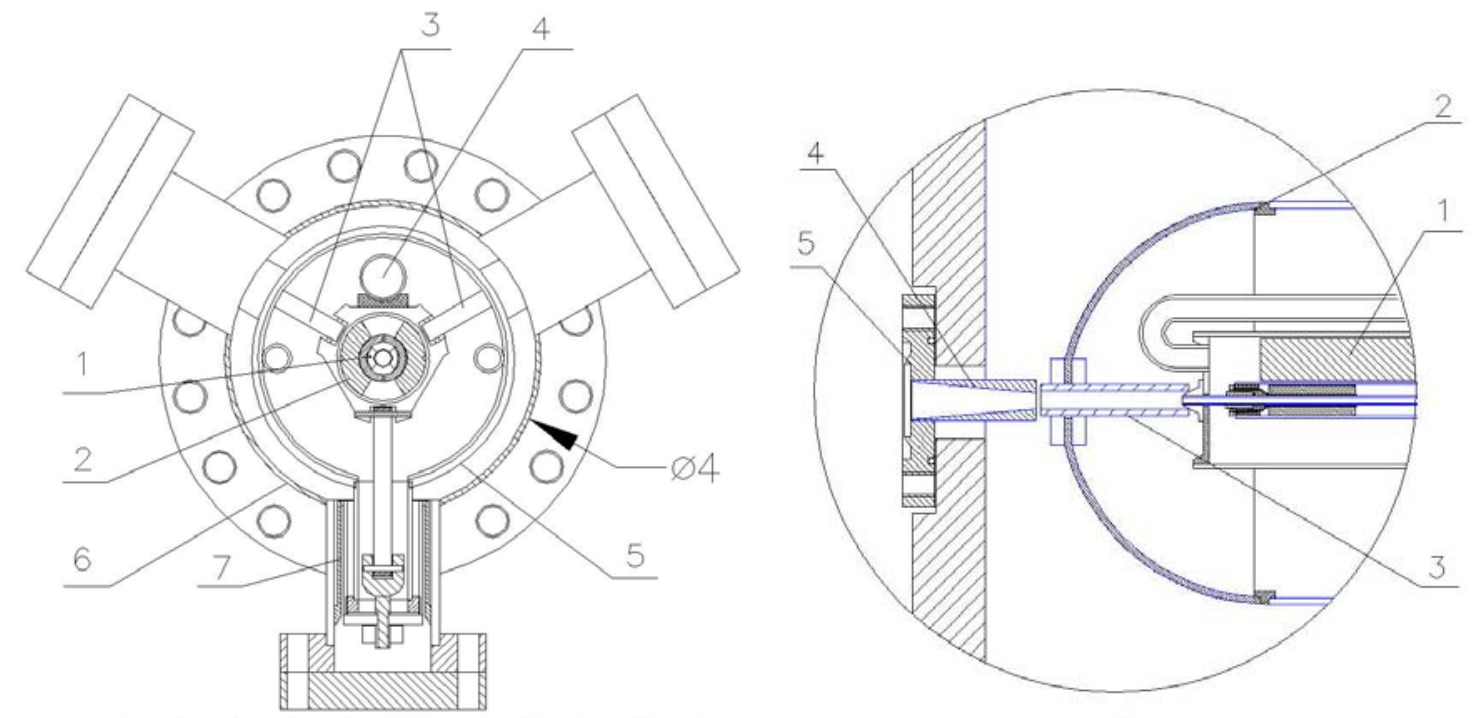

FIGURE 2: Suspension system and end of cryostat, circled in Fig.1. Left: 1-is a cold mass, 2-is a copper collar, 3are suspension straps, 4-is two phase He tube, 5 -is a $50^{\circ} \mathrm{K}$ shield, 6 -is a vacuumed corpus, 7 -is a stretching mechanism and heat lock. Right: 1 -is a cold mass, 2 -is a $76^{\circ} \mathrm{K}$-shield, 3 -is an aligning cylinder, 4-tapered diameter transformer, 5 -is a flange.

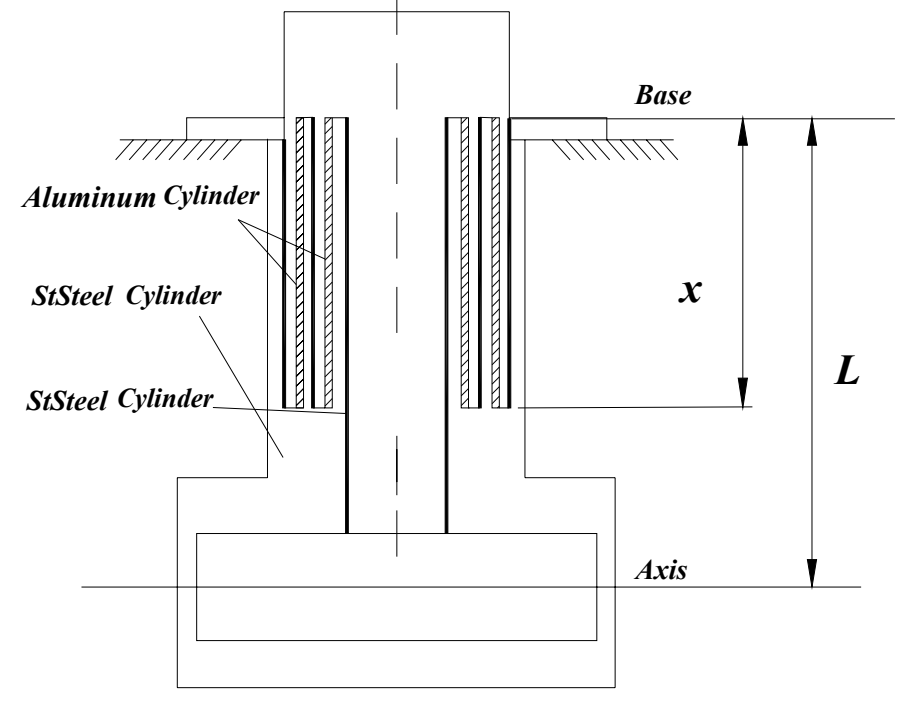

FIGURE 3: Concept of central support unit.

These suspension straps made from composite glass fibers impregnated and cured with epoxy (Fiberglass). These straps are commercially available in broad variety of sizes. 
In Fig. 3 a scheme of central support unit is represented. It arranged with few coaxial cylinders with two $\mathrm{Al}$ ones among them. For the heat flow all the length is working in series. For the lengthening the thermal expansion coefficients of $\mathrm{Al}$ and StSteel are working one against other, like in gridiron pendulum. Temperature between outer base and base of inner StSteel cylinder is distributed according to heat conductivity, practically linearly along each of cylinders. One can see from the scheme, that resulting motion of the axis of cold mass determined by equation

$$
k_{1} L \cong\left(k_{2}-\frac{1}{2} k_{1}\right) x+\left(k_{2}-\frac{1}{2} k_{1}\right) x=\left(2 k_{2}-k_{1}\right) x,
$$

where $k_{1}$ is specific lengthening of StSteel, and $k_{2}$ is specific lengthening of Al. It was taken into account, that outer (first) StSteel cylinder is operating between room temperature and $\sim 1 / 2$ of temperature of core and second one operates between $\mathrm{LN}$ and half of it. It was taken into account, that after LN temperature reached there is no practically more shrinkage, and there is no temperature gradient along $\mathrm{Al}$ cylinders. This equation (1) allow to find the distance $x$ so that there is no resulting movement of cold mass axes

$$
x \cong \frac{k_{1} L}{2 k_{2}-k_{1}} .
$$

Substitute here expressions $k_{1} \cong 3.25 \cdot 10^{-3}, k_{2} \cong 4.25 \cdot 10^{-3}$, one can obtain $x \cong 0.62 L$.

Copper collars 2 in Fig. 2 have extended area for better longitudinal heat conductivity and giving additional rigidity to the cold mass. Vacuumed corpus 6 in Fig.2 has reinforcements in region of triple-straps suspension system eliminating deformations during tensioning and movements during pumping off inner volume of cryostat.

Total heat losses calculated remains formally below $0.5 \mathrm{~W}$, however to get some operational margins the cryocooler with $1.5 \mathrm{~W}$ at $4.2^{\circ} \mathrm{K}$ and $40 \mathrm{~W}$ at $50^{\circ} \mathrm{K}$ chosen for testing device (Model RDK-415D from Janis Co.). Maintenance interval $\sim 10 \mathrm{kHours}$ is big enough to accomplish any experiment. As it was mentioned full-scale undulator will work with LHe supply.

\section{TESTED COLD MASS WITH 1-CM PERIOD}

As we mentioned in introduction the cold mass was tested in framework of VLEPP activity. Suggested that cooling can be arranged by flow of LH in gaps between yoke.

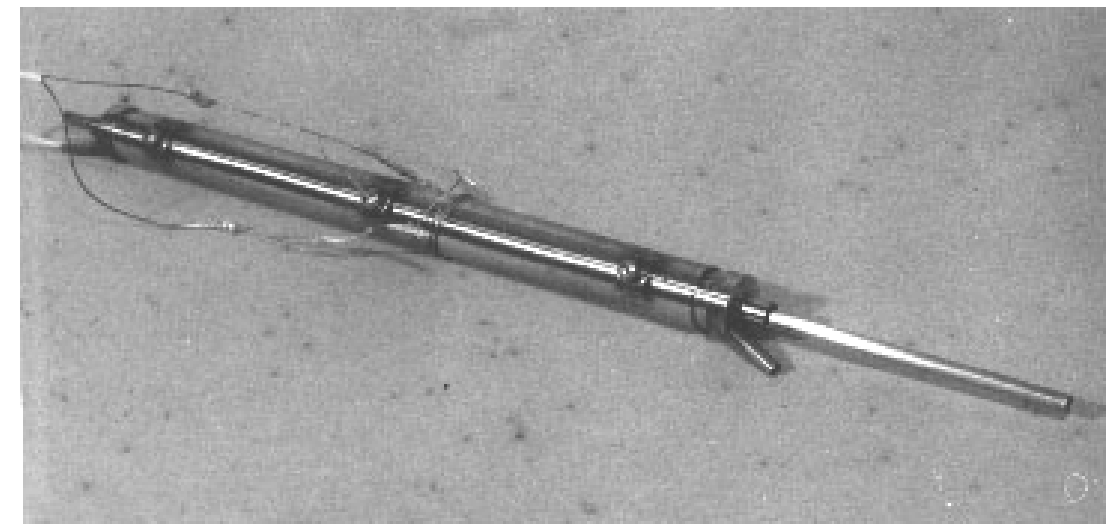

FIGURE 4: $30 \mathrm{~cm}$-long superconducting undulator with period $10 \mathrm{~mm}$ and the axis field $\sim 5 \mathrm{kG}$. Diameter of thin wall stainless steel chamber is $6 \mathrm{~mm}$, wall thickness $0.1 \mathrm{~mm}$. 

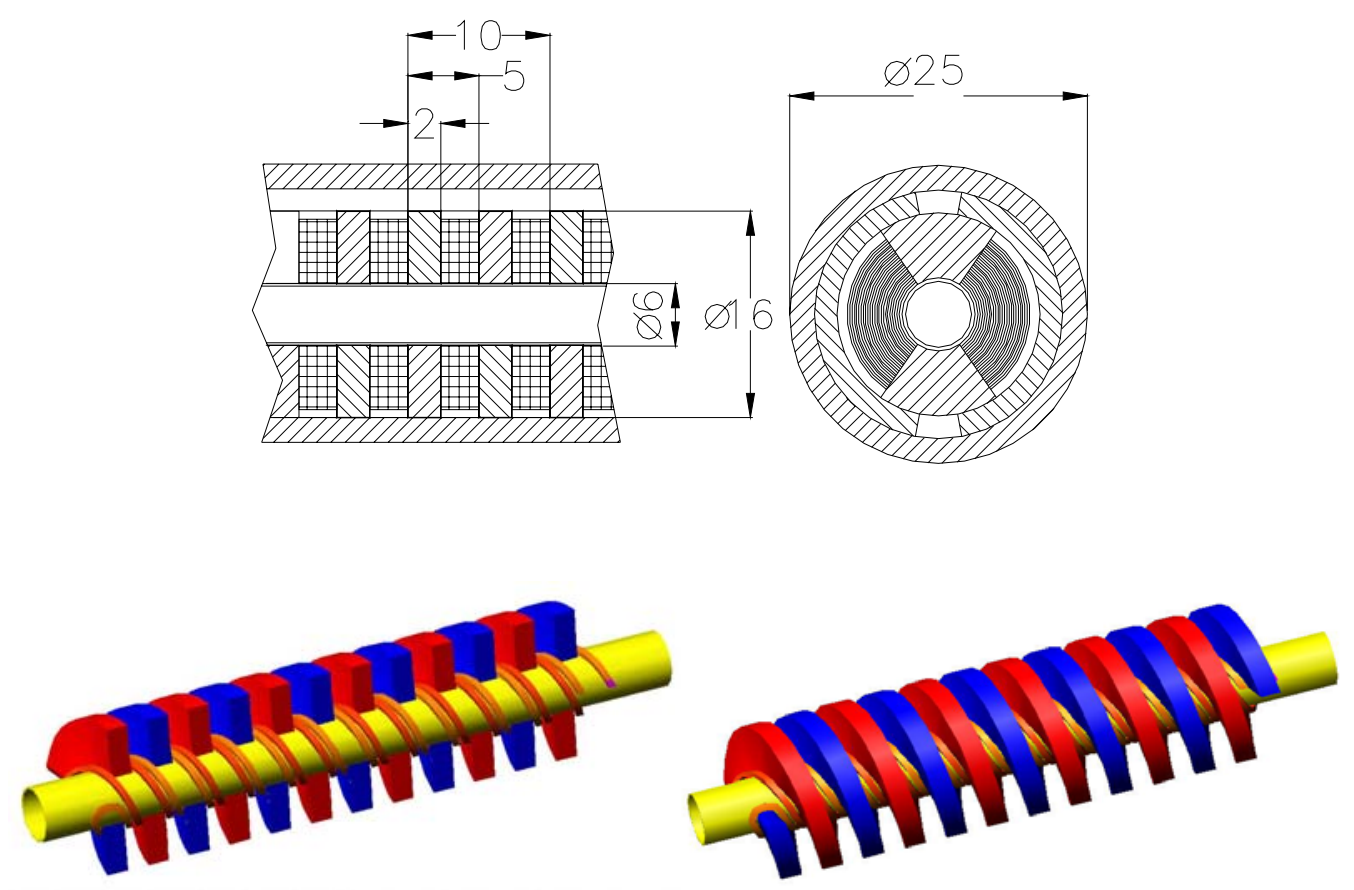

FIGURE 5: (Color) Yoke of $6 \mathrm{~mm}$ in aperture, 10-mm period of undulator with SC wires [2]. At the left vertical cut made for better look inside. Colored red and blue helical yokes made from soft steel. Half cylinders, covering yoke are not shown.

Yoke helix was machined in 10-cm long sections. After trimming these pieces soldered by Indium to the tube, so it is going without brakes.

Heat losses due to imaginary currents can be evaluated as the following. For the bunch having length $\sigma_{b}$ and number of particles $N$ peak current goes to be $\hat{I} \cong e N c / \sigma_{b}$. Resistance of section of chamber having length $L$ is

$$
R \cong \frac{L}{\sigma \cdot 2 \pi a \cdot \delta}
$$

where $a$-is inner radius of chamber, $\sigma$-is its conductivity and $\delta \cong \sqrt{\sigma_{b} / \mu_{0} \sigma c}$-is skin-depth for characteristic frequency $\omega \cong c / \sigma_{b}$. Energy dissipated by single bunch passed is going to be

$$
\hat{W} \cong \hat{I}^{2} R \frac{\sigma_{b}}{c} \cong \frac{e^{2} N^{2} c^{2}}{\sigma_{b}^{2}} \cdot \frac{L \cdot \sqrt{\mu_{0} \sigma \cdot c}}{2 \pi a \cdot \sigma \sqrt{\sigma_{b}}} \cdot \frac{\sigma_{b}}{c} \cong e^{2} N^{2}\left(\frac{c}{\sigma_{b}}\right)^{3 / 2} \frac{L}{2 \pi a} \sqrt{\frac{\mu_{0}}{\sigma}} .
$$

Substitute here $\sigma^{-1} \cong 1.7 \cdot 10^{-8} \mathrm{Ohm} \cdot \mathrm{m}, \mu_{0}=4 \pi \cdot 10^{-7} \mathrm{H} / \mathrm{m}, \quad, N=10^{10}, \sigma_{b}=0.001 \equiv 1 \mathrm{~mm}$ one can obtain $\hat{W} \cong 3.27 \cdot 10^{-6} \mathrm{~J}$. For repetition rate $f, \mathrm{~Hz}$ and for the number on bunches in train $n$, average power dissipation will be $P \cong \hat{W} \cdot f \cdot n$ Watts, remaining within $m W$ level. We used specific resistance for room temperature. As the chamber has temperature of liquid $\mathrm{He}$, this resistance must be lowered even more, at least $\sim 100$ times, making the heating by imaginary currents not serious. However this is good to test undulator operating with the beam inside in advance. If the bunch has length $\sigma_{b}=0.1 \mathrm{~mm}$ i.e. ten times shorter, the losses will be $10^{3 / 2} \cong 31$ times bigger, remaining small in absolute value. 
It was shown in [2] that thermal relaxation of core cooled by flowing Helium could be described by time and space parameters $\kappa \cong 10 \mathrm{~cm}, \tau \cong 22.4 \mathrm{~s}$ in equations describing dynamics of cooling [8]

$$
\begin{aligned}
\frac{\partial T_{H}}{\partial z} & =\frac{1}{\kappa}\left(T_{C}(z, t)-T_{H}(z, t)\right) \\
\frac{\partial T_{C}}{\partial t} & =-\frac{1}{\tau}\left(T_{C}(z, t)-T_{H}(z, t)\right),
\end{aligned}
$$

where $T_{H}$ stands for local Helium temperature, $T_{c}$ stands for local core temperature. So this is an indication of rather fast relaxation.

The wires positioned in between the yoke screw occupying area $5 \times 3 \mathrm{~mm}^{2}$. In Table 1 below the field at the axes and undulatority factor are represented as functions of total current, $\mathrm{kA} \times$ turns.

Table 1.

\begin{tabular}{|c|c|c|c|c|c|c|c|c|c|c|c|}
\hline Current,kA & 0.5 & 1 & 2 & 3 & 4 & 5 & $6^{*}$ & 7 & 8 & 9 & 10 \\
\hline$B, k G$ & 0.811 & 1.62 & 3.24 & 4.13 & 4.44 & 4.75 & 5.06 & 5.37 & 5.68 & 5.99 & 6.3 \\
\hline$K$ & 0.075 & 0.15 & 0.30 & 0.39 & 0.42 & 0.44 & 0.47 & 0.50 & 0.53 & 0.56 & 0.58 \\
\hline
\end{tabular}

Measurement in this model was carried with a help of Hall probe moved along the axis in liquid Helium. Maximal current was $\sim 6 k A \times$ turns (marked by ${ }^{*}$ ). With present day wires available on market all parameters for total current above $6 \mathrm{kA} \times$ turns in Table 1can be guaranteed.

\section{SHORT PERIOD MODEL FABRICATIONS AND TEST}

Technology tested can be scaled down to a shorter period required for possible test (or implementation, [7]) at SLAC. A six inches long model prototype with period $2.42 \mathrm{~mm}$ having all peculiarities of full length one was tested [9].

We used here the stainless steel tube of $1.5 \mathrm{~mm}$ in diameter with the wall thickness of $0.3 \mathrm{~mm}$ as a vacuum chamber. This tube was in hand at the moment. Now we are using a tube of gage size 19 with nominal OD $0.042 "(1.0668 \mathrm{~mm})$. This tube has the wall thickness of 0.0035 " $(0.0889 \mathrm{~mm})^{2}$. This tube allows the ID diameter $0.889 \mathrm{~mm}$ available for the beam. Brass, StSt or Copper tube with this diameter is available on the market.

SLAC $50 \mathrm{GeV}$ beam has emittance $\gamma \mathcal{E} \cong 3 \times 10^{-3} \mathrm{~cm} \cdot \mathrm{rad}$, which allows for the envelope function in crossover $\beta_{0} \cong 300 \mathrm{~cm}$ to have the beam size $\sigma \cong \sqrt{(\gamma \mathcal{E}) \beta_{0} / \gamma} \cong 3 \times 10^{-3} \mathrm{~cm}$. Half aperture required for ten sigma margins goes to $\cong 10 \sigma \cong 0.3 \mathrm{~mm}$ only, or $0.6 \mathrm{~mm}$ for full aperture clearance, slightly $(\sim 11 \%)$ expanding toward the ends, what is much within the tube ID.

The goals of the work done were to see if technology can be useful for scaling down all dimensions and to investigate if any sign of degradation of the wire is present, as the bending radius is small.

The winding done with pair of SC wires interlaced by soft-steel ones with the help of cylindrical director made on G10. SC wire has $0.6 \mathrm{~mm}$ in diameter- standard OXFORD 54 filament wire insulated by Formvar. The steel wires were taken with round shape and was

\footnotetext{
${ }^{2}$ New England Small Tube Catalog, tube GS\#19, XTW.
} 
annealed by direct current flow arranged with transformer, making the wire light red. The steel wires serve as a part of magnet yoke arranged by these wires and soft iron half cylinders. These cylinders surrounding the windings, shown in Fig. 2, attached to the windings by thermoconductive epoxy Epotek T-905. Undulator assembled on stock, allowing precise alignment of the inner tube during the process of squeezing and curing of epoxy. Design allows exact control of angular out-positions of the wires at the ends, and, hence, polarization of the magnetic field at the exit. The last required for proper tapering of the undulator field. Symmetric commutation with additional small iron cylinder as a magnetic shield was also tested here, Fig.6.

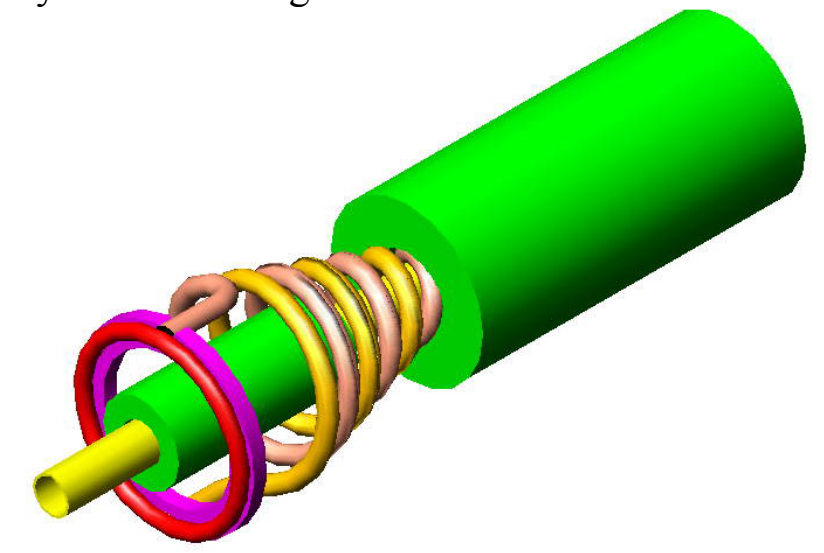

FIGURE 6: (Color) End commutation of SC wires in $2.4 \mathrm{~mm}$ model. Red ring is a $\mathrm{SC}$ wire circled and soldered with Indium to the Copper ring. Green cylinder at the right made with two halves.

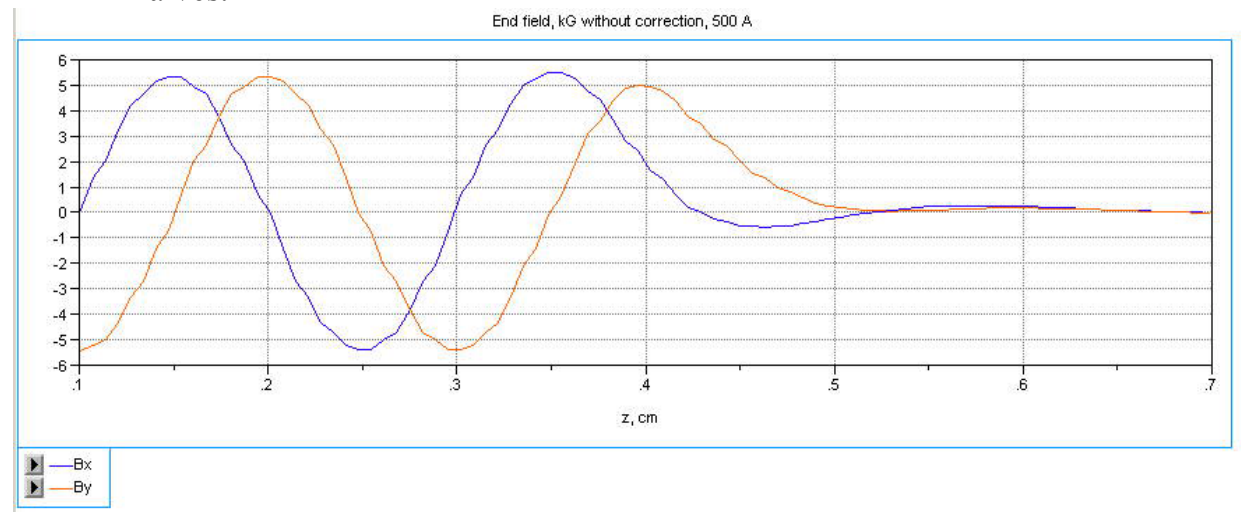

FIGURE 7: (Color) End field with conical expansion. Iron yoke ends at $0.406 \mathrm{~cm}$. Period $-2 \mathrm{~mm}$.

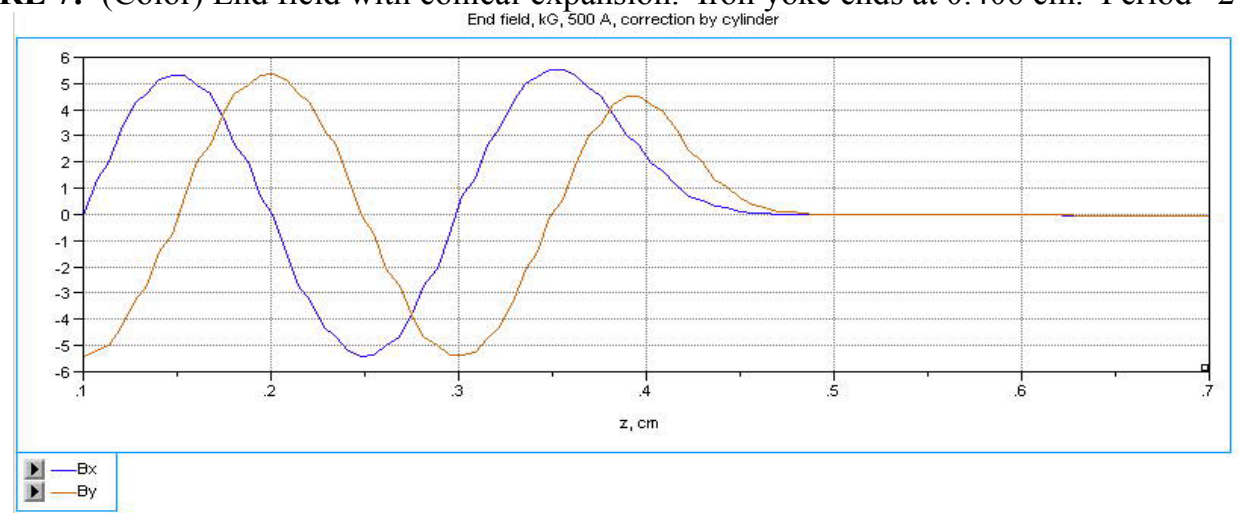

FIGURE 8: (Color) End field with conical expansion and trimming magnetic cylinder. Iron yoke ends at $0.406 \mathrm{~cm}$, same as in Fig. 7. Period $-2 \mathrm{~mm}$. 
One can see from Fig. 7 and Fig. 8, that there is some freedom to adjust the edge field with soft steel cylinders.

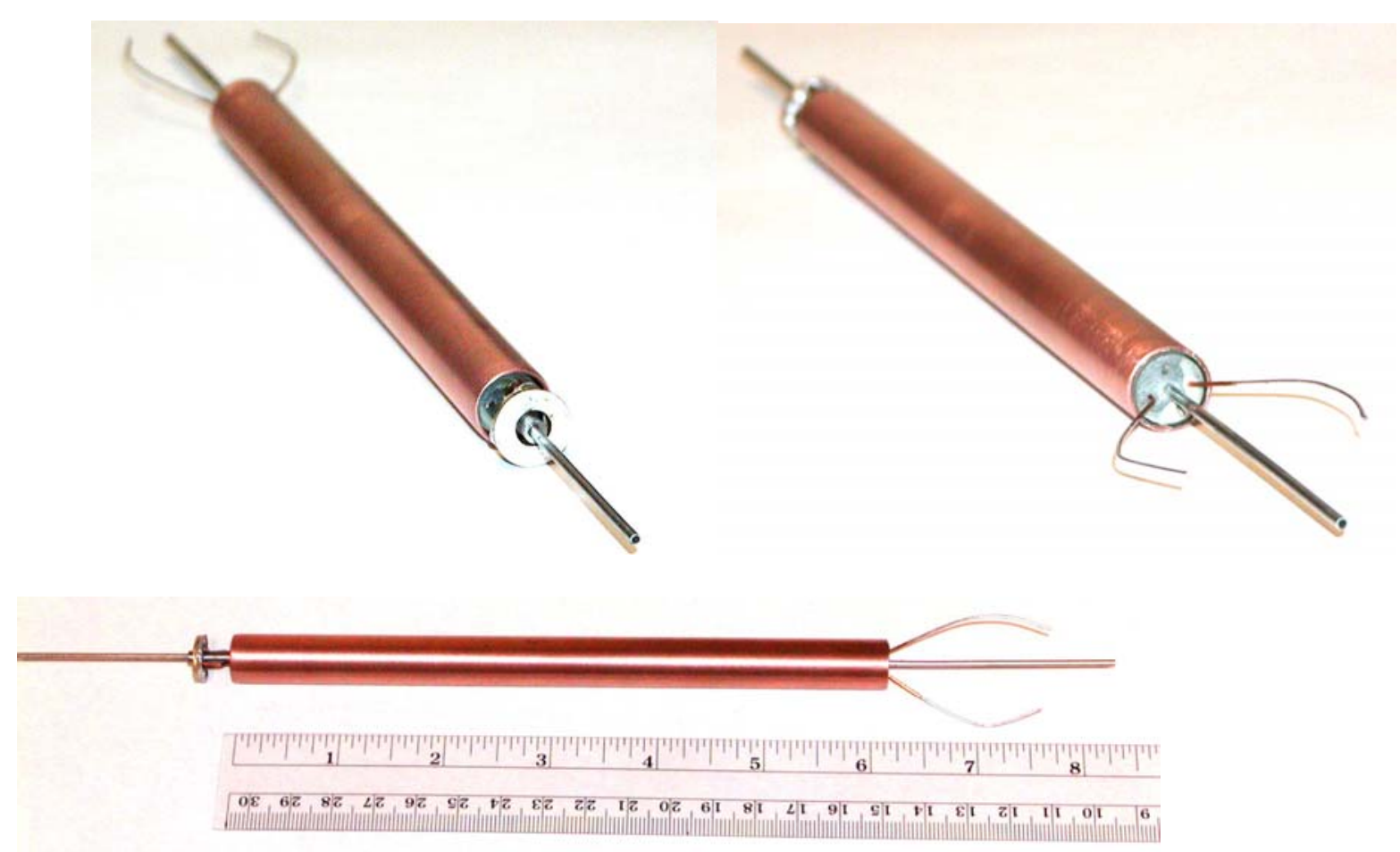

FIGURE 9: SC undulator model. Iron cylindrical shield for end trimming and ring jumper are visible here. One end of undulator has Copper jacket of $\sim 12 \mathrm{~mm}$ in diameter hides soft iron semi-cylinders having diameter $\sim 9.8$ $\mathrm{mm}$.

Undulator model was tested in a Dewar filled for the Cornell LEPP wiggler coil test set in parasitic mode, Fig.9, [9]. Pair of additional feed-through allowed independent feeding of this undulator model. No problems were found with operation of this undulator.

Field measurement in such small aperture is a problem. However the axis field module is a constant along the undulator. Measuring integrated value with scalar probe will give the value of axis field. So here the magneto-resistive sensors as a Bismuth wire can be used [10]. Micro-wire can be extruded and inserted into capillary glass-tube for mechanical rigidity and all this setup inserted into aperture. In this case as the effective region with field is full length of undulator, the variation of signal will be easy to measure and calibrate. This might be another exercise carried with this equipment.

So there was no any way to measure the field rather than to trust calculations. We estimated accuracy of correspondence of calculated to the reached one within $10 \%$. 

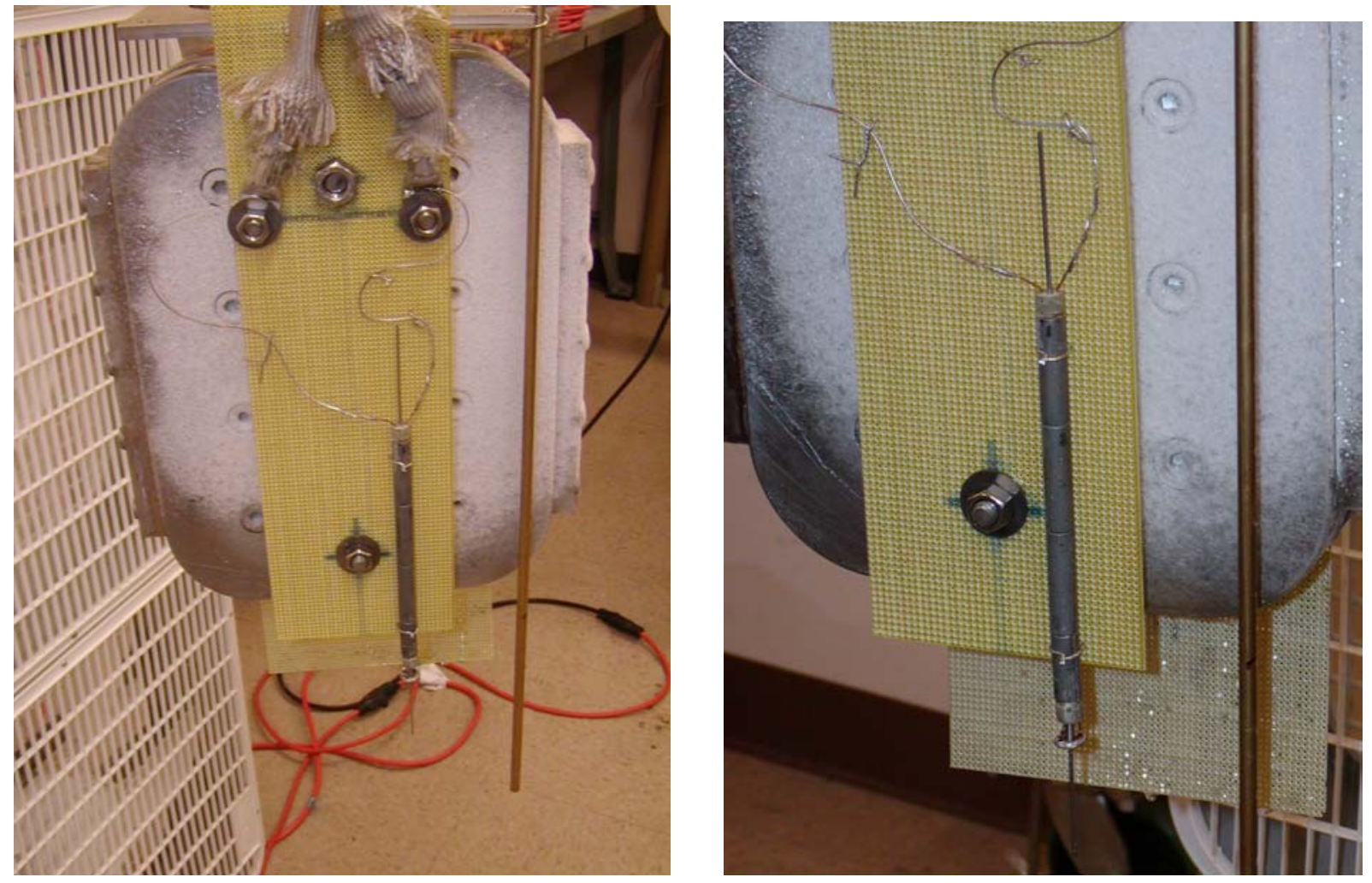

FIGURE 10: Undulator can be seen attached to the wiggler coil test fixture. Copper cover removed.

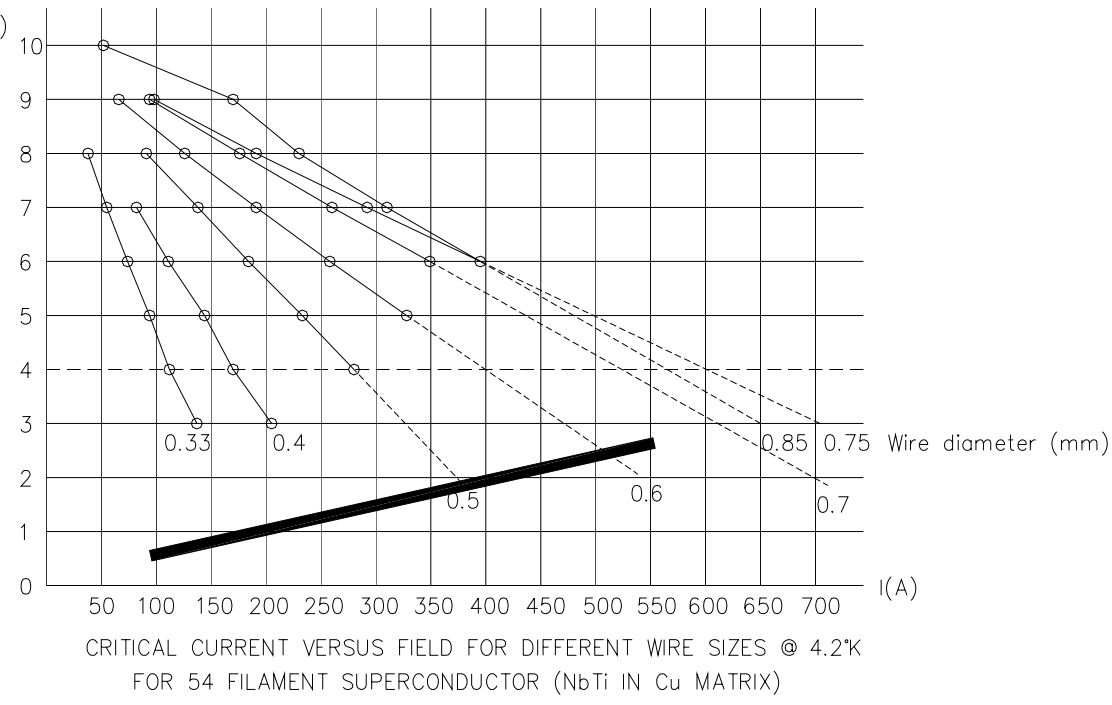

FIGURE 11: Properties of the SC wire. Loading curve for the points with highest field is represented as a solid line.

In Dewar tests we reached the current $\sim 510$ A which is close to the short sample limit, Fig.11. The field at the axis, according to calculations, reaches $\sim 0.34 \mathrm{~T}, K$ parameter, respectively, goes to $\sim 0.08$. The field amplitude between $\mathrm{SC}$ wires reaches $\sim 2.3 \mathrm{~T}$ in specific points inside the wire. The field was calculated with 3D code MERMAID. 
With the tube having $\mathrm{OD}=1.0668 \mathrm{~mm}$, as planned, parameters we expect to reach with current 500 A current the field at the axis $\sim 0.54 \mathrm{~T}$ and $K \cong 0.12$ as the field at the axis is exponentially dependent on the tube diameter. Fields between the wires remains practically the same, however. Transverse dependences of the fields are represented in Fig. 11. With additional wire layer and bit more complicated wiring undulatority factor can be increased to $K \sim 0.2$.

In final design we also are considered utilization of soft steel wire with rectangular shape, giving additional $10 \%$ to the field at the axis. The wire of this shape is available at the market. We investigated the possibility of rolling the wire of this shape from the round one in the Laboratory, however.

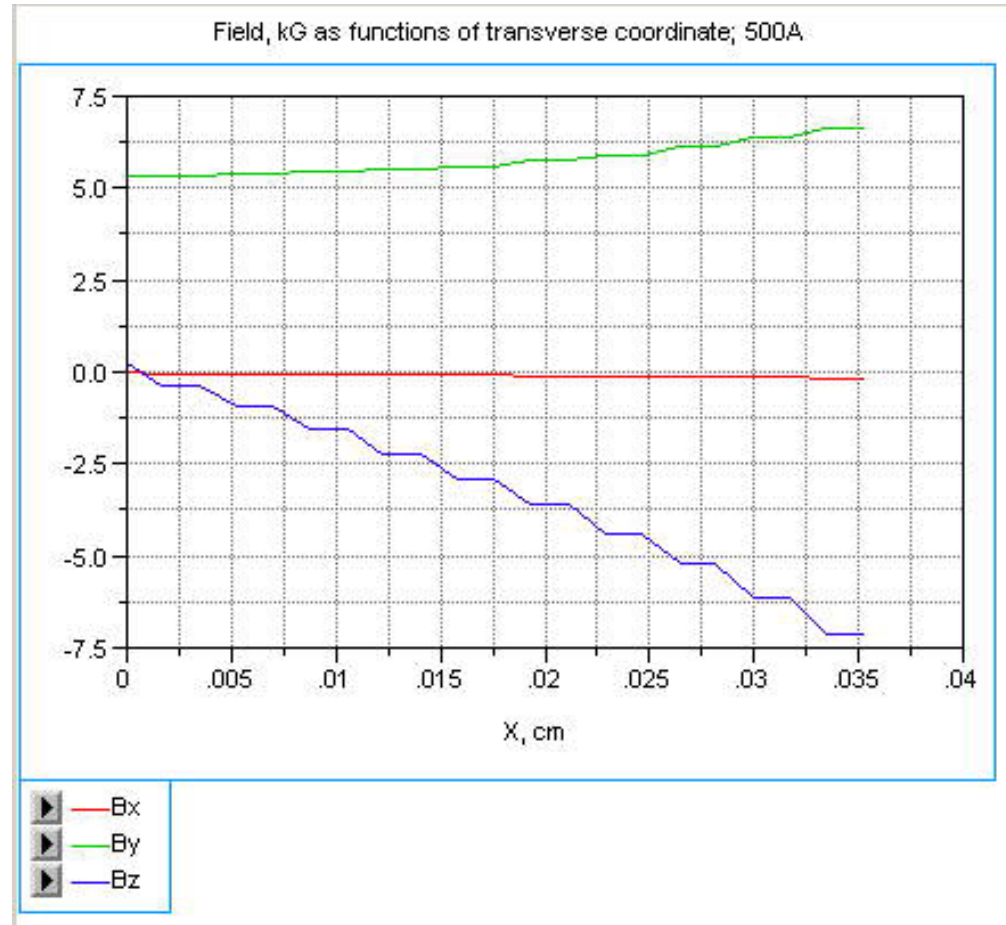

FIGURE 12: (Color) Transverse dependence of magnetic field, 500A, 2-mm period. Field in $k G, x$-in $\mathrm{cm}$. Upper curve is a vertical component; lower curve is a longitudinal one. Wires in this cross section located horizontally (along $x$ ).

Filling epoxy mixed with iron-like powder (ferrite), can also give additional $\sim 10 \%$ percent in field strength at the axis.

\section{CONCLUSIONS}

The cryostat described can accommodate any core with short-period undulator. Utilization of such SC undulator might bring significant relief in operational cost of linear collider.

Cold mass of helical SC undulator having $1-\mathrm{cm}$ period and $6 \mathrm{~mm}$ aperture clearance was successfully tested many years ago. Design can be recommended for utilization. No doubt, that technology can be successfully used for full-scale wiggler with $0.8-1 \mathrm{~cm}$ period and $\sim 100-130 \mathrm{~m}$ long.

It was shown that there are no problems with the wire in 2.4-mm period undulator. Six-inch long model of undulator cold mass with $2.4 \mathrm{~mm}$ period was manufactured and tested in Dewar.

There are no apparent limitations in scaling this technology even more down with appropriate tube and wire diameters. The aperture will go down proportionally to the period and field will 
drop exponentially, however. Period of $0.1 \mathrm{~mm}$ and $\mathrm{K} \sim 0.05$ are feasible edge values of possible design.

\section{REFERENCES}

[1] V.E. Balakin, A.A. Mikhailichenko, Conversion System for Obtaining Highly Polarized electrons and positrons, Preprint INP 79-85, Novosibirsk 1979.

V.E. Balakin, A.A. Mikhailichenko, VLEPP: the Conversion System, Proc. of the 12 Int. Conference on high energy Accelerators, Batavia, 1983, p. 127.

[2] A.A. Mikhailichenko, Dissertation, BINP, Novosibirsk, 1986, Translation: CBN 02-13, Cornell 2002.

[3] T.A. Vsevolojskaya, A.A. Mikhailichenko, E.A. Perevedentsev, G.I. Silvestrov, A.D. Cherniakin, XIII International Conference on High Energy accelerators, August 7-11, 1986, Novosibirsk.

[4] R.Pitthan, J.Sheppard, Use of Microundulators to Study Positron Production, LC02, Proceedings, SLAC-WP-21.

[5] A. Mikhailichenko, Optimized parameters of the Helical Undulator for test at SLAC, LC02, Proceedings, SLAC-WP-21.

[6] E-166, see: http://www.slac.stanford.edu/ achim/positrons/.

[7] A.A. Mikhailichenko, The feasibility of polarized $e^{+} e^{-}$collisions at SLAC B-factory, CLNS 99/1645, CORNELL U., 1999.

[8] H. Lierl, P.Shmüser, Cooldown of a HERA Octant Equipped cold Dipoles, DESY, HERA, 84/16, 1984.

[9] A. Mikhailichenko, T. Moore, First test of short period helical SC undulator prototype, Cornell CBN 02-6, June 3, 2002

[10] A.Mikhailichenko, Low aperture magnetic element measurements, (Novosibirsk, IYF). IYF89-94, Jun 1989. 12pp. (Scanned version is available at: http://ccdb3fs.kek.jp/cgibin/img/reduced_gif?198911230+1+14). 